\title{
Ion current losses in the convolute and inner magnetically insulated transmission line of the $Z$ machine
}

\author{
Eduardo M. Waisman, M. P. Desjarlais, and M. E. Cuneo \\ Sandia National Laboratories, Albuquerque, New Mexico 87185, USA
}

(Received 20 July 2018; published 19 March 2019)

\begin{abstract}
We introduce a 1D planar static model to elucidate the underlying mechanism of large ion current losses in the vacuum convolute and the inner magnetically insulated transmission line (MITL) of the $Z$ machine. We consider $\mathbf{E} \times \mathbf{B}$ electron flow, parallel to the electrodes, and ion motion across the vacuum gap, for given voltage $V$, gap distance $d$, anode magnetic field $B_{a}$, and vacuum electron current $\Delta I$. This model has been introduced and solved before by Desjarlais [Phys. Rev. Lett. 59, 2295 (1987)] for the applied magnetic field ion diode. Here we apply it to convolute and inner MITL ion losses of $Z$, relaxing the fix magnetic flux condition of that reference. In the absence of ions we show that the electron vacuum flow must be close to the anode if its current exceeds the value given by the local flow impedance, implying high electric fields there. We then introduce space charge limited ion emission from the anode, neglecting the magnetic force on ions. We obtain the solution of the steady state equations for two special cases: (a) when both the electric potential and the electric field are zero inside the gap, and there is a layer of electrons not carrying current that neutralizes the ion charge between the virtual and the electrode cathode, making that region electric field free, and (b) when the electric field is zero inside the gap, but the potential is not, and zero electron charge between that point and the physical cathode. For case (a) we obtain an ion current density which we conjecture is the maximum attainable for any electron charge distribution in the electron current carrying layer, given $V, d, B_{a}, \Delta I$ an ion species. We obtain the enhancement factor for both cases with respect to the ion-only Child-Langmuir ion current density, and show that it can be significantly larger than that of the electron saturated flow case. Furthermore, imposing electron current conservation as the flow enters the inner MITL from the four outer MITLs, we recover the well-known dependence $j_{\text {ion }} \sim V^{3 / 2} / d^{2}$, where voltage and gap are taken near the joining point of those outer MITLs. The implications and limitations of the proposed model are discussed.
\end{abstract}

DOI: 10.1103/PhysRevAccelBeams.22.030402

\section{INTRODUCTION}

The $Z$ machine is a large pulsed power accelerator at Sandia National Laboratories; its outer diameter is $33 \mathrm{~m}$. It has been amply described in the literature, for instance for a complete description of the $Z$ machine see Refs. [1,2].

We focus in this paper on the section of the $Z$ machine extending to approximately $10 \mathrm{~cm}$ from the accelerator axis shown schematically in Fig. 1. This is the section of $Z$ where it is believed that most of the machine current losses, and correspondingly power loss, occur. The region we concentrate on has cylindrical 3D periodic geometry. The four magnetically insulated transmission lines (outer MITLs) of approximately $3 \mathrm{~m}$ diameter, converge at the post-hole convolute of $Z$, located at about $10 \mathrm{~cm}$ from the

Published by the American Physical Society under the terms of the Creative Commons Attribution 4.0 International license. Further distribution of this work must maintain attribution to the author(s) and the published article's title, journal citation, and DOI. axis, where their respective currents are added into a single MITL. This single 2D radially convergent MITL, extending from the inner part of the convolute to the load, is called the inner MITL. Depicted in Fig. 1 are the four MITLs, A, B, C, D, (oftentimes referred to as levels) as they join in the convolute.

There is experimental and simulation evidence [3-6] of current loss in the vacuum convolute and in the inner MITL of $Z$ [7]. The current losses in the convolute region (CR) itself are measured directly as the difference of the sum of the outer MITL currents entering the convolute, and the current measured by Bdot probes, located at $6 \mathrm{~cm}$ from the axis [8]. The measurement of inner MITL losses is most of the time inferred from simulations when the load behavior is assumed to be known [6], or in some cases when use of techniques such as VISAR [9], allow for a direct unfold of the load current. Circuit calculations employing a detailed circuit model of the entire $Z$ machine, including physics-based load and electron and ion current loss in vacuum models [10], point to the necessity of assuming large ion current losses in the 


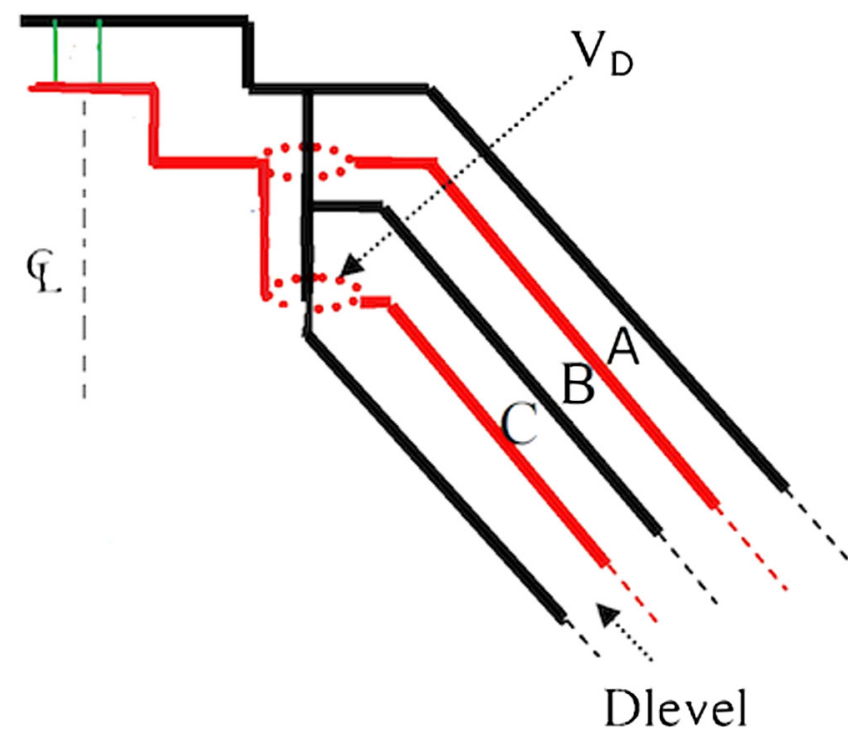

FIG. 1. Schematics of the four vacuum transmission lines (truncated) of the $Z$ machine, denominated levels A, B, C, and D. Anodes are represented by black lines and cathodes by red ones. Currents for the four levels are added up at the post-hole convolute (cathode holes shown in dotted red circles, and anode posts in black). To the left of the post-hole convolute there is a single inner MITL, and the green lines represent the load. $V_{D}$ stands for the voltage of one of the post-hole structures at level D. The convolute region goes from about 7 to $11 \mathrm{~cm}$ in radius. The vacuum transmission lines extend to a diameter of approximately $3 \mathrm{~m}$. See Refs. [1,2] for detailed descriptions.

CR and inner MITL, to adequately reproduce the electrical measurements.

In this work we only address losses caused by positive ions crossing the anode-cathode gap. Other loss mechanisms related to the possible presence of negative ions are considered in [11]. Magnetically insulated ion flow not resulting in positive ion current losses, studied for instance in Refs. [12-14], is neither part of this research.

Electron flow in the outer MITLs is well understood [15]. Less clear is where the electron charge and current go as the MITLs join into the vacuum convolute.

For the large current of the $Z$ experiments, magnetic diffusion and electron deposition heats the anode inner MITL and convolute surfaces to temperature above $400^{\circ} \mathrm{C}$ [16], resulting in neutral desorption and anode plasma formation, which is the source of ion space charge limited emission considered in this paper.

\section{PLANAR E $\times$ B FLOW IN STEADY STATE}

We assume that in order to highlight the main ion current loss mechanisms, the cylindrical system can be approximated by planar geometry; with the electric field in the negative across the gap $z$ direction, the magnetic field in the negative $y$ direction and the space electron flow in the $\mathbf{E} \times \mathbf{B}$ negative $x$ direction, which is also the direction of

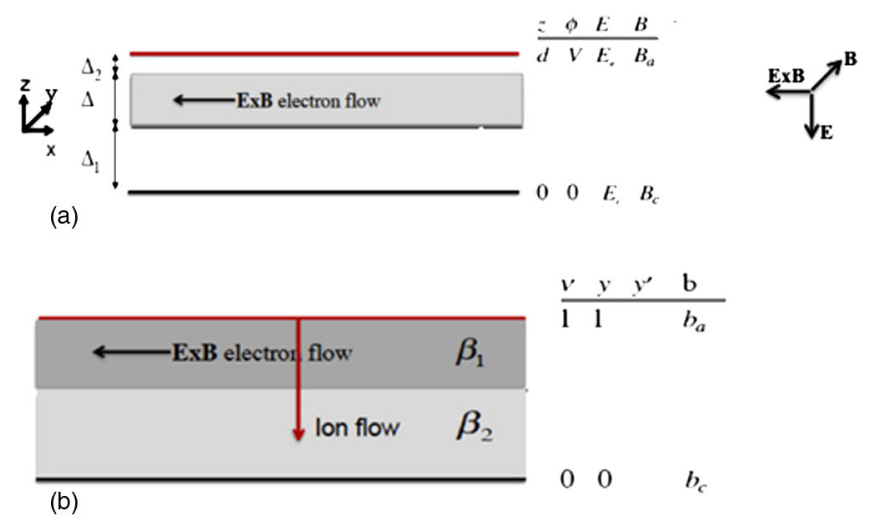

FIG. 2. (a) 1D planar electron only flow, showing coordinates and boundary conditions, as well as the directions of the fields and the electron and power flow direction $\mathbf{E} \times \mathbf{B}$. (b) Same 1D planar geometry as in (a) for the case of two electron layers, electron and ion crossing the gap, for the model we are considering; see the text for the meaning of the symbols.

the power flow into the load as shown in Fig. 2. In order to make contact with the actual convergent geometry of $Z$, we introduce the relationship $B_{a}[G]=I_{a}[A] / 5 r[\mathrm{~cm}]$, which is the relationship between the magnitude of the anode magnetic field, the anode current and the actual radius of the considered point on the $Z$ machine.

We expect that even if using a planar static 1D model is a gross oversimplification, the model will be able to point to a mechanism that may be dominant in the actual 3D geometry of the CR, and the 2D of the MITL, as well as being a guiding way to look at the actual time dependent reality.

The equations for steady electron $\mathbf{E} \times \mathbf{B}$ planar flow and cross ion current, shown in Fig. 2(a), are (neglecting the effect of the ion current on the magnetic field and vice versa)

$$
\begin{gathered}
d E / d z=4 \pi e\left(Z n-n_{e}\right), \quad \mathbf{E}=E \hat{z} \\
d B / d z=4 \pi e w_{e} / c, \quad \mathbf{B}=B \hat{y}
\end{gathered}
$$

In Eq. (1) $n$, and $n_{e}$ are the ion and electron charge number densities, and $w_{e}$ the electron velocity, while the ion charge is $e Z$, with $-e$ being the electron charge. The ion mass is $m=\mu m_{p}$, where $m_{p}$ is the proton mass. The second equality in Eq. (1b) is obtained employing the laminar $\mathbf{E} \times \mathbf{B}$ electron flow assumption, i.e., $\mathbf{w}_{\mathbf{e}}=\mathbf{E} \times \mathbf{B} / B^{2}=-w_{e} \hat{x}=-(E / B) c \hat{x}$. Unless we explicitly state different units we are using CGSGaussian units throughout. Equation (1) neglects magnetic field effects on ions, we therefore only consider in this work ion species neither magnetically significantly deflected nor insulated. 


\section{A. Electron only flow}

We first consider the case $n=0$ everywhere in the gap. From Eqs. (1a) and (1b) we immediately obtain the known pressure balance condition [17]:

$$
\begin{aligned}
E_{a}^{2}-E_{c}^{2} & =B_{a}^{2}-B_{c}^{2}=\left(I_{a}^{2}-I_{c}^{2}\right) /\left(25 r^{2}\right) \\
& \cong 2 I_{a}\left(I_{a}-I_{c}\right) /\left(25 r^{2}\right),
\end{aligned}
$$

where $I_{a}, I_{c}$ are the anode and cathode currents, respectively.

The units of Eq. (2) are CGS-Gaussian, with the exception of the currents that are in Amperes. The last approximate equality of the equation is valid for the small ratio of $\left(I_{a}-I_{c}\right) / I_{a}$. We have introduced the radius $r$ to relate to the actual radially convergent geometry of the $Z$ machine. As seen in Fig. 2(a), $E_{a}, E_{c}$ are the values of the electric field at the top and bottom edges of the electron flow, as well as at the physical anode and cathode, respectively, while $B_{a}, B_{c}$ are the values of the magnetic field at anode and cathode, respectively. Both $E$ and $B$ are constant between the edges of the electron flow strip and the respective metallic electrodes.

From Eq. (2) it follows that $E_{a}^{2} \geq B_{a}^{2}-B_{c}^{2}$. For instance, at $r=4 \mathrm{~cm}, I_{a}=20 \mathrm{MA}, I_{a}-I_{c}=1 \mathrm{MA}$, and therefore, $B_{a}=1 \mathrm{MG}, \quad B_{c}=0.95 \mathrm{MG}, \quad$ it follows that $\left|E_{a}\right| \geq$ $3.1 \times 10^{5} \mathrm{stat} \mathrm{V} / \mathrm{cm}=94 \mathrm{MV} / \mathrm{cm}$.

We assume that the electron motion is laminar and that the electrons are all going in the same power flow direction towards small radius. Thus, in the planar geometry we describe in the Introduction, the power flow is in the $\hat{x}$ direction, the magnetic field in the $\hat{y}$ direction and the electric field in the $\hat{z}$ direction. Thus the $\mathbf{E} \times \mathbf{B}$ flow is the power flow $\hat{x}$ direction. The assumption of the electron motion being towards the load in the $\hat{x}$ direction is paramount to assuming that the electric field does not change sign from cathode to anode, $E=-\phi^{\prime} \leq 0$, and thus the electric potential $\phi$ increases from cathode $z=0$ to anode $z=d$. We therefore have, for constant $n_{e}$ in the electron flow strip,

$$
-E_{a} \Delta_{2} \leq V=-E_{a}\left(\Delta_{2}+\Delta / 2\right)-E_{c}\left(\Delta_{1}+\Delta / 2\right) .
$$

The quantitiesa $\Delta_{2}, \Delta, \Delta_{1}$ are, respectively, the distance between the anode and the electron flow edge, the thickness of the electron beam, and the distance between the lower edge of the flow and the cathode per Fig. 2(a), thus $d=\Delta_{1}+\Delta+\Delta_{2}$.

For example, using the same values for $B_{a}=1 \mathrm{MG}$, $B_{c}=0.95 \mathrm{MG}$, a voltage $V=3 \mathrm{MV}$ would result in $\Delta_{2}+\Delta / 2 \leq 3.2 \times 10^{-2} \mathrm{~cm}$. That is, the upper edge of the laminar electron flow must be very near the anode. This would result in a very high electric field. We remark that there is no loss of generality in assuming constant electron density; neither is taking $\Delta_{2}=0$ as a special case within the laminar flow approximation. This latter point is consistent with the small electron Larmor radius; for the example we have given it would be of the order of $5.8 \times 10^{-3} \mathrm{~cm}$ classically and about twice of this value relativistically.

\section{B. Space charge limited ion current in cross $\mathbf{E} \times \mathbf{B}$ electrons flow}

We now turn to solving Eqs. (1a) and (1b), together with the boundary condition $E(d)=0$, voltage $V$, magnetic field $B_{a}, B_{c}$ at the electrodes, gap $d$. We assume that ions emitted at the anode cross the gap without being affected by the magnetic field. Neglecting this effect implies that there is no ion current in the power flow direction [see Fig. 2(b)].

In steady state the ion current density is constant, i.e.,

$$
\mathbf{j}_{i}=-j_{i} \hat{z} ; \quad j_{i}=Z_{e n w_{i}}=Z e n \sqrt{2 e Z(V-\phi) / m},
$$

where $w_{i}$ is the ion velocity in the $z$ direction, and the last equality in Eq. (4) comes from the ion energy conservation relation: $Z e \phi+m w_{i}^{2} / 2=Z e V$ and from setting $w_{i}(d)=0$. From Eq. (4) we have

$$
Z e n_{i}=j_{i} / \sqrt{2 e Z(V-\phi) / m} .
$$

From Eqs. (1a), (1b), and (4) we obtain

$$
B_{a}{ }^{2}-B^{2}=-E^{2}+16 \pi j_{i}\left(\sqrt{V-\phi} /(2 e Z / m)^{1 / 2} .\right.
$$

To obtain the result of Eq. (6) we have invoked $\phi(d)=V, E(d)=E_{a}=0$.

If we further assume the presence of a "full virtual cathode," setting $\phi=0, E=0$, in Eq. (6) at a given point inside the gap, $0<z_{0}<d$, we obtain a particular value of the ion current density $j_{0}$ :

$$
j_{0}=\left[\left(B_{a}^{2}-B_{c}^{2}\right) / \sqrt{V}\right](2 e Z / m)^{1 / 2} / 16 \pi,
$$

Recalling that $B_{a}^{2}-B_{c}^{2}=2 \Delta I \bar{I} / 25 r^{2}, I[A], B[G]$, where $\Delta I=I_{a}-I_{c}$, and $2 \bar{I}=I_{a}+I_{c}$, we can rewrite Eq. (7a) employing units $V, A, A / \mathrm{cm}^{2}, \mathrm{~cm}$ :

$$
j_{0}\left[A / \mathrm{cm}^{2}\right]=2.20 \times 10^{-4}(Z / \mu)^{1 / 2} \Delta I \bar{I} /\left(\sqrt{V} r^{2}\right) .
$$

This current density is realized, given the boundary conditions we have stated, for a special case we will discuss below. We will see that it requires sufficient electron charge per unit area to be realized. That is, given $V, B_{a}, d, Z, \mu$ and a diamagnetic drop from $B_{a}$ to $B_{c}$, assuming the presence of a virtual cathode determines both its position and the electron charge in the gap.

We recall that the space charge limited ion-only Child-Langmuir (CL) current density is given by $j_{\mathrm{CL}}=$ $(2 e Z / m)^{1 / 2} V^{3 / 2} / 9 \pi d^{2}$, and we will use this as a 
normalization factor for the actual $j_{i}$, defining the enhancement factor $\eta=j_{i} / j_{C L}$.

We now convert our differential equations (1) to dimensionless variables: $\varphi=\phi / V, \nu=z / d$. In terms of these variables the equation for the dimensionless potential $\varphi$ is given by

$$
\varphi^{\prime \prime}(\nu)=\alpha / \sqrt{1-\varphi(\nu)}-\beta(\nu) .
$$

In Eq. (8) we have defined $\alpha \equiv(4 / 9) j_{i} / j_{C L}$, $\beta \equiv 4 \pi n_{e} e d^{2} / V$. Thus, the enhancement factor is $\eta=9 \alpha / 4$. On the other hand, $\alpha=4 / 9, \beta=0$ corresponds to the ion-only CL solution. The boundary conditions for Eq. (8) are, $\varphi(1)=1, \varphi(0)=0, \varphi^{\prime}(1)=0$. Furthermore, the condition that a given electron current produces a diamagnetic effect reducing the magnetic field from $B(\nu)$ to $B_{c}$ becomes

$$
\left(b^{2}-b_{c}^{2}\right) / 2=\int_{0}^{v} d \nu \beta(\nu) \varphi^{\prime}(\nu),
$$

where $b^{2}=B^{2} d^{2} / V^{2}$, and in particular,

$$
\gamma_{0}^{2} \equiv\left(b_{a}^{2}-b_{c}^{2}\right)=2 \int_{0}^{1} d \nu \beta(\nu) \varphi^{\prime}(\nu) .
$$

There are infinite nondenumerable solutions for Eqs. (8) and (9), depending on the boundary conditions and the electron density distribution $\beta(\nu)$. We restrict the solution set by not allowing the electric field to change sign, precluding counterflow of electrons, which in terms of the derivative of the scaled potential becomes $\varphi^{\prime} \geq 0$. We also consider only piecewise constant electron charge density, that is, $\beta$ is piecewise constant.

We first seek solutions for which there is a virtual cathode, i.e., $\varphi^{\prime}\left(\nu_{0}\right)=0,0 \leq \nu_{0}<1[18,19]$. The motivation for considering $\beta$ piecewise constant is that it allows us to solve the governing Eqs. (8) and (9) analytically, while not being excessively restrictive, we can always think of the constant $\beta$ representing the average electron charge density over the region considered.

The motivation for allowing a virtual cathode is that, for given voltage, magnetic field, gap distance and space electron flow current, we believe that this provides the maximum possible enhancement. We show in a later section that there is a choice of electron density distribution for which this ion current enhancement is realized.

Multiplying both sides of Eq. (8) by $\varphi^{\prime}$ and integrating from $\nu_{2} \geq \nu \geq \nu_{1}$ we obtain

$$
\left.\varphi^{\prime 2}\right|_{\nu_{1}} ^{\nu_{2}}=\left.[4 \alpha \sqrt{1-\varphi}-2 \beta(1-\varphi)]\right|_{\nu_{1}} ^{\nu_{2}}
$$

Changing variables in Eq. (10a) by defining $u=\sqrt{1-\varphi}$, we obtain

$$
\left.\left(u^{\prime} u\right)^{2}\right|_{\nu_{1}} ^{\nu_{2}}=\left.\left(\alpha u-\beta u^{2} / 2\right)\right|_{\nu_{1}} ^{\nu_{2}}
$$

For the upper region $1 \geq \nu \geq \nu_{0}$ from Eq. (10a) recalling $u^{\prime}(1)=0$ we have

$$
u^{\prime}=-\sqrt{(2 \alpha-\beta u) / 2 u} .
$$

We integrate Eq. (11) to obtain, in the upper region, $1 \geq \nu \geq \nu_{0}$,

$$
\nu=[\sqrt{u(q-u)}-q \arcsin \sqrt{u / q}] \sqrt{2 / \beta}+1,
$$

where $q=2 \alpha / \beta, \quad \beta \neq 0$.

The solution exhibited in Eq. (12) has been obtained before (Ref. [20]), where they were applied to magnetically insulated ion diodes. We remark that since we are imposing $\varphi^{\prime} \geq 0 \Rightarrow u^{\prime} \leq 0$ that the inverse function $\varphi(\nu)$ is unique.

We can also solve Eq. (10) for $\beta=0$ in the lower region i.e., the interval $0 \leq \nu \leq \nu_{0}$. In the special case $u^{\prime}\left(\nu_{0}\right)=\varphi^{\prime}\left(\nu_{0}\right)=0$, we obtain

$\nu=\nu_{0}-(2 / 3)\left(2 u_{0}+u\right) \sqrt{u-u_{0}} / \sqrt{\alpha}, \quad u_{0} \equiv u\left(\nu_{0}\right)$.

We will treat two distinct cases (high enhancement or HE, and low enhancement or LE). We impose continuity of potential and electric field and allow for discontinuous charge density.

\section{High enhancement}

We consider electron density $\beta_{1}$ constant for $\nu_{0} \leq \nu \leq 1$, followed by a different constant $\beta_{2}$ for $0 \leq \nu \leq \nu_{0}$, producing a full virtual cathode. We will call this case high enhancement (HE), because we will show that enhancement factors for this case are potentially high. This is precisely the model solved by Desjarlais, for the so-called $\rho=1$ case in Refs. $[18,19]$. The main difference of this work with that reference, is that there the magnetic flux is constant; while in our case we do not apply this restriction. We also have solved directly using the magnetic field instead of the magnetic potential, both approaches being completely equivalent. As shown in Eqs. (12) and (13), an analytic solution of $\nu(\varphi)$ can be obtained, which as we mentioned before, is an invertible function. We show in Appendix B the equivalence of this approach with the solution of Refs. [18,19] for $\rho=1$ (using the nomenclature of those papers). 


\section{Low enhancement}

In this case, no electron charge is allowed beyond the diamagnetic electron current carrying layer, relaxing the condition that the point of zero electric field is also the point where the potential is zero. We call this case low enhancement (LE), because we show that the enhancement factor can only attain a maximum limit we derive.

In the sections that follow, we describe the solution for these two cases. We neglect electron emission at the physical cathode, which is a good approximation because the emitted electron sheath electrostatically shields it, carrying a negligible amount of the total electron space current.

\section{High enhancement solution}

In Eq. (10a) the scaled potential satisfies the conditions $\varphi(1)=1, \quad \varphi^{\prime}(1)=0$. By further requiring $\varphi\left(\nu_{0}\right)=\varphi^{\prime}\left(\nu_{0}\right)=0$, it follows that $\beta_{1}=2 \alpha$. It is also immediately obvious from Eq. (8) that by making the total dimensionless charge density $\varphi^{\prime \prime}=0$, for $0 \leq \nu \leq \nu_{0}, \varphi, \varphi^{\prime}$ stay zero for the same interval, from which it follows that $\beta_{2}=\alpha=\beta_{1} / 2$. That is, the electron charge density in that charge-neutral region is one half of the charge density in the electron current carrying layer, and it is the same as the ion charge density there. It is also evident, given the choice of boundary conditions for this case, that the ion current density $j_{0}$ of Eq. (7) is attained, and that from Eq. (9), and from $\beta_{1}=2 \alpha$,

$$
\begin{gathered}
\beta_{2}=\alpha=\beta_{1} / 2=\gamma_{0}^{2} / 4 \\
\eta=(9 / 4) \alpha=(9 / 16) \gamma_{0}^{2} \\
\delta=1-\nu_{0}=\pi / \gamma_{0} .
\end{gathered}
$$

We remark that, as anticipated when we introduced $j_{0}$, Eq. (14) shows the relationship between having a given diamagnetic effect represented by $\gamma_{0}$, and the enhancement factor $\eta$. They also give the necessary electron charge $\beta_{1}$, $\beta_{2}$, as well as the dimensionless distance between anode and virtual cathode, $\delta$. The voltage and gap distance appear in the scaling factors used to define the dimensionless variables utilized in those equations.

Also, as expected, Eq. (14) implies that the larger the enhancement factor the larger the electron charge density necessary in the upper and lower layers, to carry the electron current and to neutralize the ion charge density in the lower layer, respectively.

We notice that for the HE case, as seen from our derivation of Eq. (7a), it is not necessary to assume constant electron charge density in the upper region to attain the ion current density $j_{0}$, as long as there is a neutral lower layer characterized by $\beta_{2}=\gamma_{0}^{2} / 4$, and that the dimensionless charge distribution is such as to produce the same diamagnetic drop, i.e., $\int_{\nu_{0}}^{1} \beta_{1}(\nu) \varphi^{\prime}(\nu) d \nu=\gamma_{0}^{2} / 2$.
We conjecture that for given values of anode and cathode magnetic field, voltage, gap, and total electron charge per unit area, $j_{0}$ given by Eq. (7) and realized in this HE case, is the highest attainable ion current density within the model we are considering.

One particular such solution is the one for which $\nu_{0}=0$. This is the so-called electron saturated flow, introduced in Refs. $[19,20]$. From Eq. (14) setting $\nu_{0}=0$ we obtain

$$
\begin{aligned}
\pi / 2 & =\sqrt{\beta / 2} \Rightarrow \beta=\pi^{2} / 2, \quad \alpha=\pi^{2} / 4, \\
\eta & =(9 / 4) \alpha=9 \pi^{2} / 16=5.55 .
\end{aligned}
$$

The value for the enhancement factor $\eta$ in Eq. (15) is exactly what was found in those references for the ion current density for this particular saturated electron flow case.

It is also interesting to notice that this HE case behaves as if only the upper electron layer existed, see Refs. [18,19]. From the point of view of the circuit quantities there would be no way to differentiate between having a physical or rather a virtual cathode. In fact, it can be shown, by straightforward algebra, that

$$
\begin{aligned}
j_{0} & =\left(9 \pi^{2} / 16\right) j_{C L}(V, \delta d) \\
& =\left(9 \pi^{2} / 16\right)(2 e Z / m)^{1 / 2} V^{3 / 2} /(\delta d)^{2}
\end{aligned}
$$

That is, $j_{0}$ is the saturated ion current density for the diode formed between the anode and the virtual cathode.

Thus, the dependence of the enhancement factor is a function, at a given voltage, ion type and gap distance, of $B_{a}^{2}-B_{c}^{2}=2 \Delta I \bar{I} / 25 r^{2}$, where $\bar{I}=\left(I_{a}+I_{c}\right) / 2$, when assuming sufficient electron charge to carry the diamagnetic current, and to neutralize the ion charge density beyond that layer. In fact there is a one to one relationship between charge per unit length and the electron diamagnetic current, which can be derived straightforwardly from Eq. (14):

$$
\begin{aligned}
q_{e} & =(r V / 8 d)\left(\gamma_{0}^{2}+\pi \gamma_{0}\right) \\
& =\left[\Delta I I_{a} d /(100 r V)\right]+\pi \sqrt{2 \Delta I I_{a}} / 40,
\end{aligned}
$$

where in Eq. (16) we have set $B_{a}^{2}-B_{c}^{2}=2 \Delta I \bar{I} / 25 r^{2}$ and assumed $I_{a} \gg \Delta I$.

Therefore to realize HE as expressed in Eq. (16) and its corresponding enhancement factor of Eq. (14b), the charge per unit length should be sufficient so that $\gamma_{0} \geq \pi$.

From these considerations we infer that "the valve" determining the ion current enhancement factor is the electron space current magnitude and distribution entering the convolute and inner MITL region, coming from the outer MITLs without striking the anode.

We show in Figs. 3-6 relevant quantities for this case, for input corresponding to the following example, we call EX: $C^{+}, V=3 \mathrm{MV}, d=0.5 \mathrm{~cm}, I_{a}=20 \mathrm{MA}$, $r=4 \mathrm{~cm}$. For instance, for $\Delta I=0.4 \mathrm{MA}$ EX yields an 


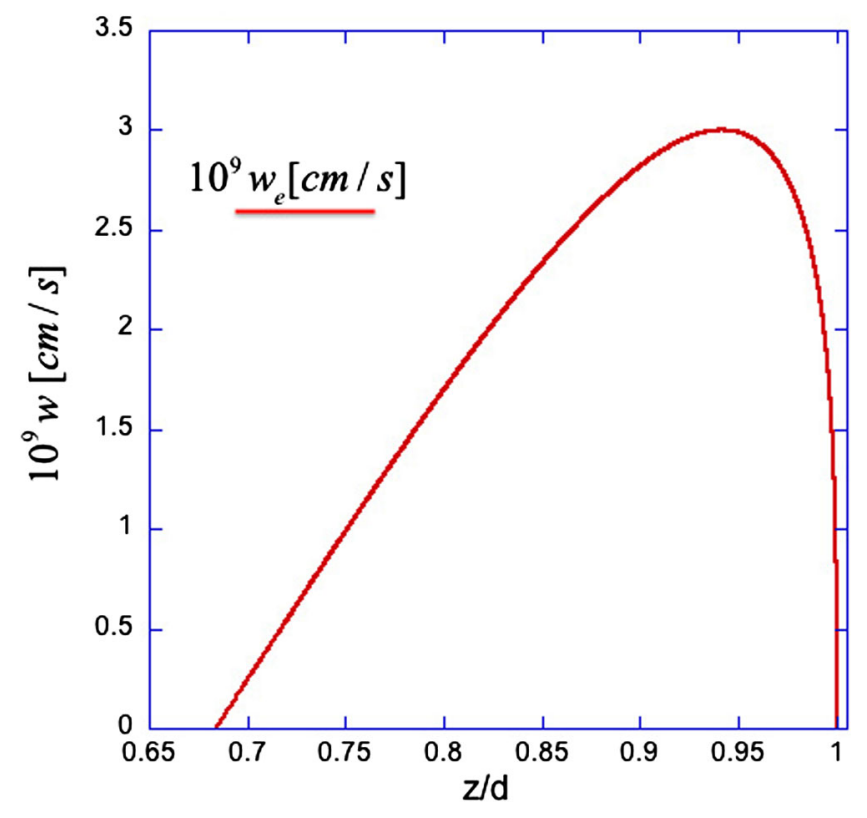

FIG. 3. Electron velocity in units of $10^{9} \mathrm{~cm} / \mathrm{s}$ in the electron current carrying layer for EX in the HE regime of Sec. II B 3, corresponding to $\Delta I=0.4 \mathrm{MA}$. See the text for the meaning of the symbols.

enhancement factor of $\eta=55.7$, yielding the $C^{+}$current density $j_{i}=1.82 \times 10^{4} \mathrm{~A} / \mathrm{cm}^{2}$.

\section{Low enhancement solution}

For this case we start with a given value of $\alpha$ and find a value of $\beta$ for the upper layer setting $u^{\prime}\left(\nu_{0}\right)=0$,

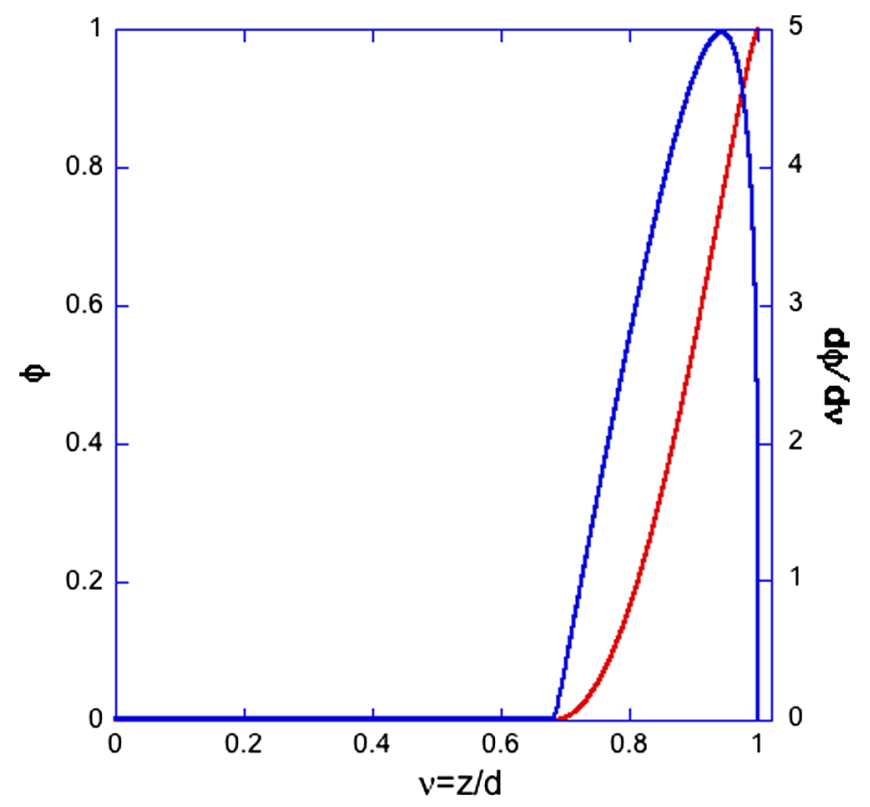

FIG. 4. Dimensionless potential (red, left scale) and electric field magnitude. The electric field (blue, right scale) peak is about $5 \mathrm{~V} / \mathrm{d}, 30 \mathrm{MV} / \mathrm{cm}, \mathrm{EX}$ for the HE regime of Sec. II B 3 and $\Delta I=0.4$ MA. See the text for the meaning of the symbols.

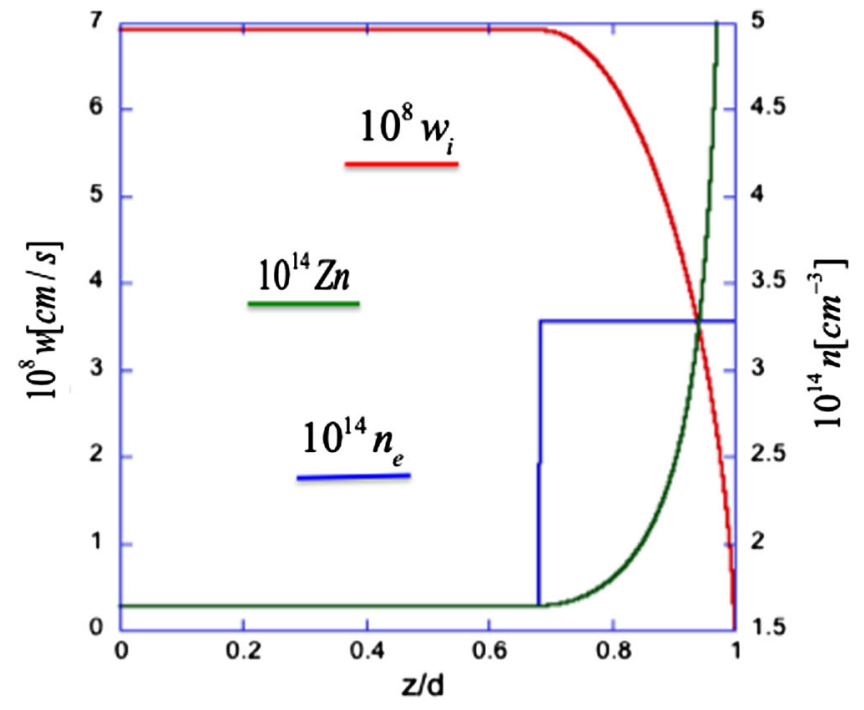

FIG. 5. Ion and electron number densities profiles (right scale), for $\mathrm{EX}$ in the HE regime of Sec. II B 3, and $\Delta I=0.4 \mathrm{MA}$. The ion number density in units of $10^{14} \mathrm{~cm}^{-3}$ (green), which goes to infinity at the anode (integrable singularity) is cutoff to allow comparison with the electron number density (blue), in the same units as electron number density. The ion velocity multiplied in units of $10^{8} \mathrm{~cm} / \mathrm{s}$ (left scale) is shown in red.

$u\left(\nu_{0}\right)=u_{0} \leq 1$ in Eq. (12). In this manner a selected value of $\alpha$ determines the values of $\beta$, and $u_{0}, \nu_{0}$, and thus from Eq. (9b) the magnitude of the diamagnetic effect,

$$
\left(B_{a}^{2}-B_{c}^{2}\right)(d / V)^{2} / 2 \equiv \gamma_{0}^{2} / 2=\beta\left(1-\varphi_{0}\right)=\beta u_{0}^{2} .
$$

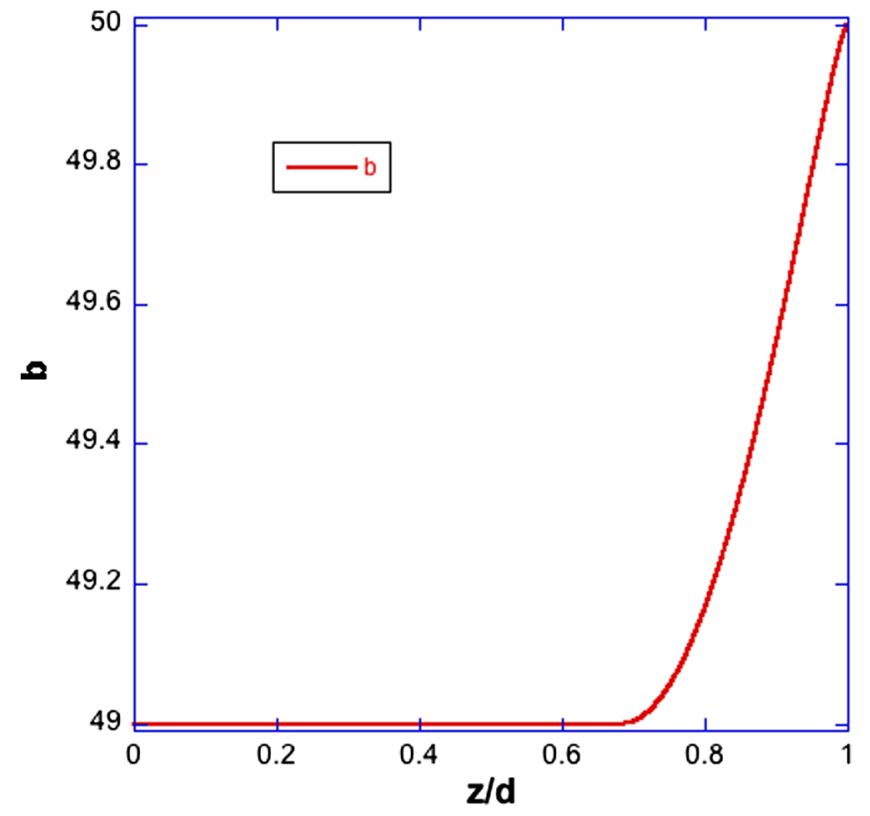

FIG. 6. Profile of dimensionless magnetic field for EX in the $\mathrm{HE}$ regime of Sec. II B 3, and $\Delta I=0.4 \mathrm{MA}$. See the text for the meaning of the symbols. 


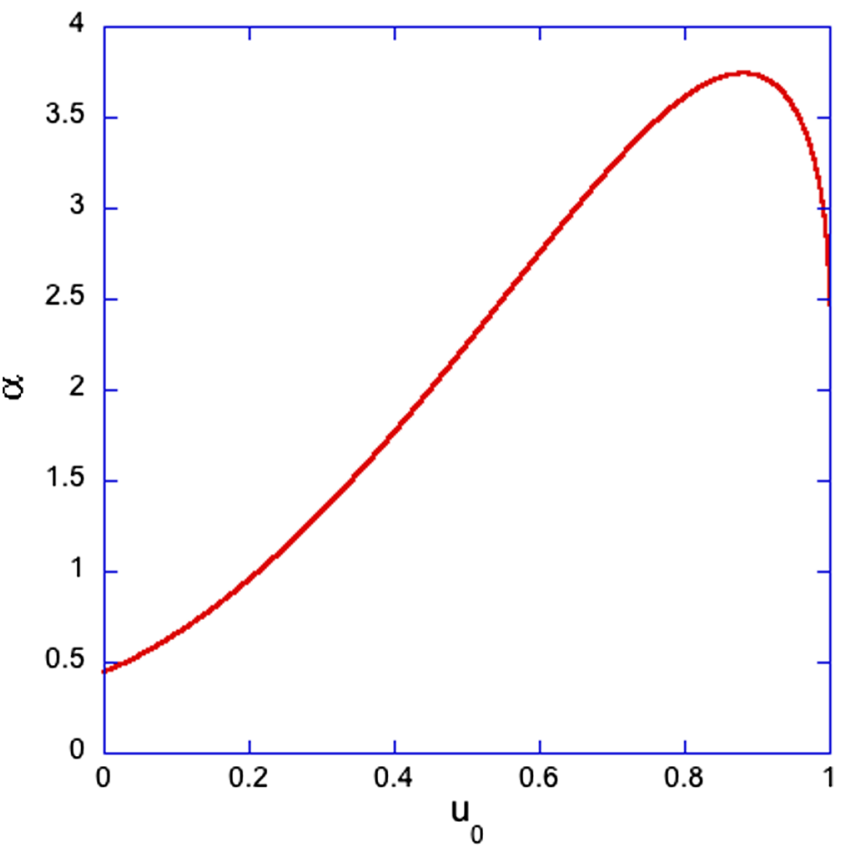

FIG. 7. Value of $\alpha\left(u_{0}\right)$, where $u_{0}=\sqrt{1-\varphi_{0}}$, where $\varphi_{0}$ the value of the dimensionless potential at the point that the electric field is zero. See the text for the meaning of the symbols in Sec. II B 4.

From Eq. (11), making $u^{\prime}\left(\nu_{0}\right)=0$, implies that $\beta u_{0}=2 \alpha, q=u_{0}$. Using this relationship in Eq. (12) we obtain

$$
\sqrt{\alpha} \nu_{0}=\sqrt{\alpha}-u_{0}^{3 / 2} \pi / 2
$$

and from Eq. (13), applicable for no electron charge density in the lower layer, setting $u(0)=1$, it follows that

$$
(2 / 3)\left(1+2 u_{0}\right) \sqrt{u-u_{0}}=\sqrt{\alpha} \nu_{0}
$$

Thus, from Eqs. (18) and (19) we find

$$
\sqrt{\alpha}=(2 / 3)\left(1+2 u_{0}\right) \sqrt{1-u_{0}}+u_{0}^{3 / 2} \pi / 2
$$

In Fig. 7 we plot $\alpha=f\left(u_{0}\right)$. Clearly given a value of $\alpha$ we can obtain the corresponding value of $u_{0}$ and vice versa, and from it $\beta=2 \alpha / u_{0}$, and all other relevant quantities. The most important feature of Eq. (20), as seen in Fig. 7, is that there is a maximum attainable value $\alpha=\alpha_{\max }=$ 3.7408 , which corresponds to a maximum enhancement factor of $\eta=8.417$.

This is the maximum attainable enhancement for LE. We also have found, as expected, that for $u_{0}=0, \alpha=4 / 9$.

For this LE case we show in Fig. $8 \varphi(\nu)$ and $\varphi^{\prime}(\nu)$ for $\alpha=\alpha_{\text {max }}$, respectively.

For the same values of anode magnetic field, voltage, gap, and radius of EX we find that $\Delta I=52.8 \mathrm{kA}$ and $j_{i}=2747 \mathrm{~A} / \mathrm{cm}^{2}$. Remarkably, it takes a relatively small

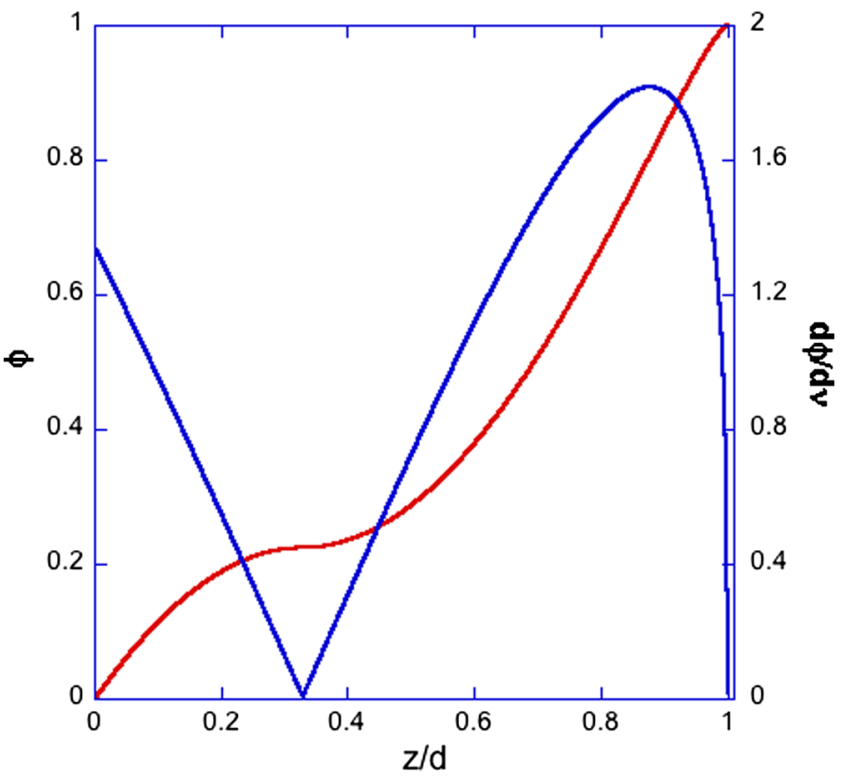

FIG. 8. Dimensionless potential (red, left scale) and its derivative (blue, right scale) for $\alpha_{\max }$ LE regime of Sec. II B. For LE the value of $\Delta I$ is determined by the function plot in Fig. 7, for EX $\Delta I=52.8 \mathrm{kA}$, for $\alpha=\alpha_{\max }=3.7408$. See the text for the meaning of the symbols.

electron flow current, about $0.26 \%$ of the anode current, to achieve this maximum enhancement factor.

For $u_{0}=1$, i.e., $\varphi_{0}=0$, we recover the electron flow saturated case $\alpha_{\text {sat }}=(\pi / 2)^{2}, \eta_{\text {sat }}=5.55$. We also remark that for $\alpha_{\text {sat }} \leq \alpha<\alpha_{\max }$, where $\alpha_{\text {sat }}=2.468$, there are two possible values of $u_{0}$ and thus two possible values of the diamagnetic effect value, $\gamma_{0}^{2}=2 \beta u_{0}^{2}=4 \alpha u_{0}, 9.87 \leq \gamma_{0}^{2} \leq$ 13.62 corresponding to two distinct values of the dimensionless electron charge density $\beta$ (see Fig. 7).

As in the HE case a relation between charge per unit length and electron diamagnetic current is easily calculated, in this LE case numerically.

We conclude this section by remarking that the cases shown are, as mentioned before, just examples of electron charge density distributions. However, the solutions we have derived provide indeed a rationale for a plausible existence of large enhancement factors of the nonmagnetically insulated ion current density with respect to the iononly CL case.

The actual electron density distribution is determined by the dynamics of the electron flow entering the $Z$ convolute and inner MITL region from the outer MITLs, and it can be best studied by detailed 3D codes such as CHICAGO [21]. It is important to notice that in this model that actual electron space charge and current in the outer MITLs determine the ion losses in the inner MITL and convolute. This fact should be used to suggest validation experiments and further simulations, and if confirmed would be paramount in the future design of larger pulsed power machines beyond $Z$. 


\section{HEURISTIC SCALING FOR ION LOSSES IN THE INNER $Z$ MITL}

We recover a CL-like relation, if we assume the ion current density given in Eq. (7), at times near the peak current, when the voltage at the outer MITLs near the convolute is approximately the same as the voltage in the inner MITL. We can write for any of the four levels (see Fig. 1 and captions for meaning of levels and Refs. [1,2]), in practical units, i.e., $V, A, \Omega$,

$$
I_{a i}^{2}-I_{c i}^{2}=V^{2} / Z_{f i}^{2}, \quad Z_{f i} \simeq 30 d_{i} / r_{i}, \quad i=1,4,
$$

where $Z_{f}(\Omega)$ is defined in Ref. [17].

By noting that $I_{a i}^{2}-I_{c i}^{2}=\left(I_{a i}+I_{c i}\right)\left(I_{a i}-I_{c i}\right) \simeq$ $2 I_{a i} \Delta I_{i} \simeq 2\left(I_{a} / 4\right)(\Delta I / 4)$, where $I_{a}, \Delta I$ are the total anode and electron space current entering the convolute, respectively, we can write

$$
\left.\left(I_{a}^{2}-I_{c}^{2}\right) \simeq \sum_{i=1}^{4}\left(I_{a i}^{2}-I_{c i}^{2}\right)=16 \overline{\left(I_{a i}^{2}-I_{c i}^{2}\right)}=16 V^{2} / \overline{\left(Z_{f i}\right.}\right)^{2} .
$$

We substitute this result of Eq. (22) in Eq. (7b) obtaining

$\left.j_{0}\left[A / \mathrm{cm}^{2}\right]=1.76 \times 10^{-3}(Z / \mu)^{1 / 2} V^{3 / 2} /\left[\overline{\left(Z_{f i}\right.}\right)^{2} r^{2}\right]$.

We express $\left.\overline{\left(Z_{f i}\right.}\right)^{2}=(30 \bar{d} / \bar{r})^{2}$ with the result

$j_{0}\left[A / \mathrm{cm}^{2}\right]=1.96 \times 10^{-6}(Z / \mu)^{1 / 2}\left(V^{3 / 2} / \overline{d^{2}}\right)(\bar{r} / r)^{2}$.

The enhancement factor with respect to the "average" CL ion current density, with voltage and gap distance corresponding to their average values taken at the outer MITLs convolute joining point, is thus

$$
\eta=36(\bar{r} / r)^{2}
$$

For instance, for $C^{+}, V=3 \mathrm{MV}, \bar{d}=0.6 \mathrm{~cm}, \bar{r}=7 \mathrm{~cm}$, $r=5 \mathrm{~cm}$ we obtain $j=1.2 \times 10^{4} \mathrm{~A} / \mathrm{cm}^{2}$, an enhancement factor of 70.6.

Clearly the CL-like dependence applies because of the assumption that the electron flow generated in the outer MITLs goes through the convolute without any of its current being lost to the anode before the radial position considered in Eqs. (23) and (24). Thus, the enhancement we have derived in Eq. (24) should be an upper limit.

For the time in the pulse when the voltage is approximately constant along the "inductance line" $(\dot{I} \simeq 0$ for purely inductive loads) we have

$$
\begin{aligned}
I_{\text {ion }}[A] & =\int_{r_{\min }}^{r_{\max }} 2 \pi j_{0} r d r \\
& =1.23 \times 10^{-5}\left(V^{3 / 2} / \bar{d}^{2}\right) \sqrt{Z / \mu} \bar{r}^{2} \ln \left(r_{\max } / r_{\min }\right) .
\end{aligned}
$$

The maximum and minimum radii of Eq. (25) are approximately the convolute radius, and a radius close to the load, respectively. Since the ion current in Eq. (25) depends logarithmically on their ratio, we can take for example that ratio to be, say 5. For the example EX suggested above, we would therefore have $I_{\text {ion }} \simeq 3$ MA. We remark that these large ion losses result in some of the ion current going in the direction of the power flow, thus in turn modifying the structure of the magnetic field. We have neglected this, as it is expected not to be a dominant effect in our search for the main underlying mechanisms of ion losses in the region of $Z$ under consideration.

\section{CONCLUSIONS}

We have been able to solve analytically in relevant cases the 1D steady state equations in planar geometry, for ion anode limited space charge current in the presence of an electron layer undergoing laminar $\mathbf{E} \times \mathbf{B}$ flow. We have introduced this simplified model in search of an underlying mechanism to elucidate current losses in the CR and inner MITL of the $Z$ machine experiments. In fact those experiments are actually time dependent, and the experimental arrangement corresponds to cylindrical 3D periodic geometry at the convolute of $Z$, and $2 \mathrm{D}$ convergent geometry elsewhere in $Z$, see Fig. 1. We found the possibility, within this simple steady state 1D planar model, of high enhancement factors for the ion current density over the ion-only CL solution, for relatively small diamagnetic electron current. We distinguished two cases: low and high enhancement, for two-layer piecewise constant electron charge density, depending on the values of the charge density on those layers. This applies to high voltages and high enough magnetic fields, as to make the $\mathbf{E} \times \mathbf{B}$ flow assumption valid, and for ion species not magnetically insulated, and heavy enough to be able to neglect the magnetic field force on them.

In the examples we have given in this paper we have considered $\mathrm{C}^{+}$, rather than $\mathrm{H}^{+}$as the heavy ion, because for the magnetic fields, voltage and anode-cathode gaps of those examples protons are magnetically insulated, while $\mathrm{C}^{+}$or for example $\mathrm{O}^{+}$are not. For the values we employed in the examples, $V=3 \mathrm{MV}, B=1 \mathrm{MG}, d=0.5 \mathrm{~cm}$, the proton Larmor radius is $0.25 \mathrm{~cm}$, and instead it is $0.87 \mathrm{~cm}$ for $\mathrm{C}^{+}$and $1.0 \mathrm{~cm}$ for $\mathrm{O}^{+}$.

We give the examples of those ions because water and hydrocarbon desorption appear to be the main source of the anode plasma, see Ref. [22] and references therein. 
However, protons would not be magnetically insulated when voltage and magnetic field are such that the proton Larmor radius, $r_{\text {proton }}[\mathrm{cm}]=2.5 \times 10^{3} \sqrt{V[\text { stat } V]} / B[G] \geq$ $g(t)$, is larger than the effective anode-cathode gap. This effective gap results from both electrodes plasma expansion. Moreover, for situations such as the HE regime described in this paper, the effective gap would be determined from the edge of the expanding anode plasma and the virtual cathode, beyond which and up to the expanding cathode plasma the electric field is zero and the electric potential constant.

It is completely straightforward to do the relevant calculations, using the models offered in this paper to then compute the proton loss.

We remark that in dynamic calculations which ions cross the gap depends on whether they are assumed to be available in large enough densities in the anode plasma for space charge limited emission to occur, and, as stated above, on their local time dependent Larmor radius being larger than the effective anode-cathode gap.

We conclude that either we have large enhancement factors over CL, or there is no steady state situation like we describe coming from the actual dynamics. That is, the presence of electron $\mathbf{E} \times \mathbf{B}$ flow of such magnitude as we found necessary to provide those large enhancement factors might be impossible. We could discern this by performing idealized CHICAGO calculations with very high resolution. For instance, we could do a 2D calculation at constant voltage and prescribed magnetic fields at the electrodes, where in a cathode anode gap a beam of electrons is injected. We would need very high resolution and small time steps, of the order of $10^{-3} \mathrm{~cm}, 10^{-14} \mathrm{~s}$. Such a calculation would clarify questions of instabilities, convergence and actual electron charge density distribution.

Although there has been in the past several years ground breaking LSP particle in cell code 3D simulations, in steady state [23], and time dependent [24], of the $Z$ convolute and part of the inner MITL they were not geared to investigate ion losses other than protons. The minimum radius simulated was $4 \mathrm{~cm}$. They were understandably performed with relatively low resolution, of the order of $1 \mathrm{~mm}$ cells. Moreover, to the best of our understanding they do not contain analysis of, for instance, what is the local ion loss at a given location "reading" the voltage and anode magnetic field there, which is what would be needed for a direct comparison between the simplified model presented in this work and those simulations. This is the reason why we suggest well-resolved calculations including smaller radii to elucidate whether the mechanisms described here are present in those numerical simulations we propose. We believe that understanding of main mechanisms can only enhance the design and interpretation of future numerical simulations.

We have given a heuristic argument showing how to recover a CL-like solution for the ion current density, depending on the actual flow entering the gap from the outer MITLs into the convolute and inner MITL of $Z$. We show that the commanding factor is the average flow impedance of the outer MITLs, determining the electron flow input. We calculated the enhancement factor over the ion only CL solution approximately.

The consequences of the finding of this model and our heuristic arguments, assuming they happen to capture the main mechanism of ion losses in the post-convolute region, are as follows.

1. The high electric fields produced by the electron flow in the absence of ion charge in that gap could perhaps be the cause of the anode plasma creation, e.g., at electric fields of 50-100 MV/cm, field ionization of the desorbed neutral layer would be rapid. If this were physically realizable it would add a mechanism of anode plasma formation, other than Ohmic heating, electrons hitting the anode and UV [22].

2. Ion current losses in the convolute and postconvolute region depend entirely on what happens with the electron flow at the outer MITLs, before the convolute. The loss thus depends on voltage and flow impedance of the outer MITLs.

3 . The highest fractional ion losses $I_{\text {ion }} / I_{a}$ occur at high voltage and lower anode current, as shown in Eq. (25), i.e., the ion loss being proportional to $V^{3 / 2}$ and independent of the anode current.

\section{ACKNOWLEDGMENTS}

We are grateful for insightful discussions we had with Brian Hustel, and with Bill Stygar on the physics based model for the $Z$ machine. We thank Bill Stygar for suggesting that we examine consequences of having a virtual cathode, discussed in the paper. We also thank David Rose for useful discussions on the convolute simulations performed by him and his colleagues.

Sandia National Laboratories is a multimission laboratory managed and operated by National Technology and Engineering Solutions of Sandia, LLC, a wholly owned subsidiary of Honeywell International, Inc., for the U.S. Department of Energy's National Nuclear Security Administration under Contract No. DE-NA0003525. This paper describes objective technical results and analysis. Any subjective views or opinions that might be expressed in the paper do not necessarily represent the views of the U.S. Department of Energy or the United States Government.

\section{APPENDIX A: MEDIUM ENHANCEMENT CASE: TWO ELECTRON LAYERS}

We proceed to solve for the more general case in which there are two layers with different dimensionless electron charge densities (again piecewise constant), $\beta=\beta_{1}$, for $\nu_{0} \leq \nu \leq 1$, and $\beta=\beta_{2}$ for $0 \leq \nu<\nu_{0}$ and the electric field being zero inside the physical gap. Then we have from Eq. (10) for the upper and lower regions, 


$$
\begin{gathered}
u^{\prime 2}=\alpha / u-\beta_{1} u / 2, \quad \nu_{0} \leq \nu \leq 1 \\
\left(u^{\prime} u\right)^{2}=\left(\beta_{2} / 2\right)\left(-u^{2}+q_{2} u+1-\gamma_{0}^{2} / 2 \beta_{2}\right), \quad 0 \leq \nu \leq \nu_{0} .
\end{gathered}
$$

Equation (A2) satisfies the conditions $u(1)=0, u^{\prime}(1)=0$. In Eq. (A2) we have defined $q_{2}=2 \alpha / \beta_{2}$, and obtain it by imposing continuity of $u, u^{\prime}$, at $\nu_{0}$. The diamagnetic effect condition of Eq. (9a) for this present case is

$\beta_{1} u_{0}^{2}+\beta_{2}\left(1-u_{0}^{2}\right)=\gamma_{0}^{2} / 2, \quad$ where $u_{0}=u\left(\nu_{0}\right)$.

The analytic solution $\nu(u)$ for Eqs. (A1) and (A2) is then $\nu=1+\sqrt{2 / \beta_{1}}\left[\sqrt{u\left(q_{1}-u\right)}-q_{1} \arcsin \sqrt{u / q_{1}}\right]$,

for $\nu_{0} \leq \nu \leq 1$

and for $0 \leq \nu \leq \nu_{0}$ :

$$
\begin{aligned}
\nu= & \sqrt{2 / \beta_{2}}\left\{\sqrt{-u^{2}+q_{2} u+\left(1-\gamma_{0}^{2} / 2 \beta_{2}\right)}\right. \\
& \left.-\left(q_{2} / 2\right) \arcsin \left[\left(2 u-q_{2}\right) / \sqrt{\operatorname{det}}\right]\right\}+D .
\end{aligned}
$$

The constant $D$ is determined by imposing the condition $\nu(1)=0$, i.e.,

$$
\begin{aligned}
D= & \sqrt{2 / \beta_{2}}\left[-\sqrt{-1+q_{2}+\left(1-\gamma_{0}^{2} / 2 \beta_{2}\right)}\right. \\
& \left.+\left(q_{2} / 2\right) \arcsin \left(2-q_{2}\right) / \sqrt{q_{2}^{2}+4\left(1-\gamma_{0}^{2} / 2 \beta_{2}\right)}\right]
\end{aligned}
$$

where det $=q_{2}^{2} u^{2}+4\left(1-\gamma_{0}^{2} / 2 \beta_{2}\right), q_{i}=2 \alpha / \beta_{i}, i=1,2$.

The charge densities $\alpha, \beta_{1}, \beta_{2}$ must fulfill the following constraints for a solution to exist, and satisfy the boundary conditions, and the requirement that $u^{\prime} \leq 0: q_{1} \leq 1$, and $-u^{2}+q_{2} u+1-\gamma_{0}^{2} / 2 \geq 0,\left|2 u-q_{2}\right| \leq 1$, for $0 \leq \nu \leq \nu_{0}$.

A general solution would have three free parameters, for instance $\beta_{1}, \beta_{2}, u_{0}$. By requiring, as in the LE and HE cases, that $u_{0}^{\prime}=u^{\prime}\left(\nu_{0}\right)=0$, for this extra condition only two parameters are free, for instance $\beta_{1}, \beta_{2}$. From this condition from Eqs. (A1) and (A2), it follows that

$q_{1}=2 \alpha / \beta_{1}=u_{0}, \quad$ and that $-u_{0}^{2}+q_{2} u_{0}+1-\gamma_{0}^{2} / 2 \beta_{2}=0$,

respectively. We recall that to realize the HE case one can select $\beta_{1}$ and it follows that $\beta_{2}=\beta_{1} / 2$, determining all other quantities; while in the LE we can chose $\beta_{1}$ and make $\beta_{2}=0$, also determining all the other parameters, as discussed in Secs. II B 3 and II B 4 in the main text.

Applying the condition $u_{0}^{\prime}=u^{\prime}\left(\nu_{0}\right)=0$, we find from Eq. (A4) the following nonlinear algebraic equation:

$$
\begin{aligned}
\nu_{0}= & 1-\sqrt{2 / \beta_{1}}\left(q_{1} \pi / 2\right) \\
= & \sqrt{2 / \beta_{2}}\left[-\sqrt{q_{2}-\gamma_{0}^{2} / 2 \beta_{2}}\right. \\
& \left.+\frac{q_{2}}{2}\left(\sin ^{-1} \frac{\left(2-q_{2}\right)}{\sqrt{\mathrm{det}}}+\pi / 2\right)\right],
\end{aligned}
$$

where all the quantities appearing in this equation can be expressed in terms of $\beta_{1}$ and $\beta_{2}$, employing Eqs. (A3) and (A5).

From Eq. (A6), it is straightforward to verify that one obtains the HE case for $\beta_{2}=\beta_{1} / 2$, and the LE one for $\beta_{2} \rightarrow 0$.

\section{APPENDIX B: REPRODUCING DESJARLAIS $\rho=1$ CASE FROM REFS. [18,19]}

For this HE case with the virtual cathode inside the physical gap, i.e., $\varphi\left(\nu_{0}\right)=0, \varphi^{\prime}\left(\nu_{0}\right)=00<\nu_{0} \leq 1$, we have, directly from Eqs. (9) and (10),

$$
\alpha=\beta_{1} / 2, \quad \beta_{1}=\gamma_{0}^{2} / 2 .
$$

Also from Eq. (14),

$$
\nu_{0}=1-\pi / \gamma_{0} .
$$

To complete the statement of the problem we have to impose flux conservation as it was done in Refs. [18,19]. That is,

$$
F=\int_{x_{c}}^{d} B d x=B_{0} d,
$$

where in (B3), $F$ is the magnetic flux, and $B_{0}, x_{c}, d$ are the quantities defined in Ref. [19] ( $B_{0}$ here is the so-called $\bar{B}_{0}$ in that reference). Turning to the nomenclature of the present paper, and in CGS-Gaussian units,

$$
F=B_{0} d=V f=V \int_{\nu_{c}}^{1} b d \nu,
$$

$$
\text { where } b \equiv B d / V, \quad \text { and } \quad \nu=x / d .
$$

The limiting voltage can be obtained by taking the limit of the enhancement factor going to infinity, that is when the applied magnetic ion diode impedance collapses, thus, $\eta=(9 / 4) \alpha=(9 / 16) \gamma_{0}^{2} \rightarrow \infty$, or equivalently $\gamma_{0} \rightarrow \infty$, occurring as $\nu_{c} \rightarrow 1$.

Therefore,

$$
V_{*}=B_{0} d / f_{*} .
$$


To obtain the limiting dimensionless flux in the electron layer we have from Eq. (9a) in terms of the variable $u$,

$$
\begin{aligned}
f_{*} & =\lim _{\nu_{c} \rightarrow 1} \int_{\nu_{c}}^{1} b d \nu \\
& =\lim _{\nu_{c} \rightarrow 1} \int_{\nu_{c}}^{1} \sqrt{b_{c}^{2}+\gamma_{0}^{2}\left(1-u^{2}\right)} d \nu \\
& =b_{c^{*}} \lim _{\nu_{c} \rightarrow 1} \int_{\nu_{c}}^{1} \sqrt{1+\gamma_{0}^{2}\left(1-u^{2}\right) / b_{c^{*}}^{2}} d \nu,
\end{aligned}
$$

where $b_{c^{*}} d=B_{0} x_{0} /\left(d+x_{0}\right)=B_{0} / 4, x_{0}=0.5 \mathrm{~cm}, d=1.5 \mathrm{~cm}$. By making the change of variables $d \nu=d u / u^{\prime}=$ $-d u \sqrt{4 / u(1-u) / \gamma_{0}^{2}}$, which follows from Eq. (10a) and (B1), the limit of the integral of the last equality of Eq. (B6) becomes; $\int_{0}^{1} d u \sqrt{u(1+u)}=[3 \sqrt{2} / 2-\ln (3+2 \sqrt{2})]$. Thus from Eq. (B5),

$$
\begin{aligned}
b_{c^{*}} d & =B_{0} x_{0} /\left(d+x_{0}\right)=B_{0} / 4, \\
x_{0} & =0.5 \mathrm{~cm}, \quad d=1.5 \mathrm{~cm},
\end{aligned}
$$

From which it follows $V_{*}=0.595 B_{0} d$, to three significant figures, which is indeed the result of Ref. [19] for the case $\rho=1$.

[1] R. B. Spielman, C. Deeney, G. A. Chandler et al., Tungsten wire-array Z-pinch experiments at $200 \mathrm{TW}$ and $2 \mathrm{MJ}$, Phys. Plasmas 5, 2105 (1998).

[2] W. A. Stygar, P. A. Corcoran, H. C. Ives et al., 55-TW magnetically insulated transmission-line system: Design, simulations, and performance, Phys. Rev. ST Accel. Beams 12, 120401 (2009).

[3] M. R. Gomez, R. M. Gilgenbach, M. E. Cuneo, C. A. Jennings, R. D. McBride, E. M. Waisman, B. T. Hutsel, W. A. Stygar, D. V. Rose, and Y. Maron, Experimental study of current loss and plasma formation in the $\mathrm{Z}$ machine post-hole convolute, Phys. Rev. Accel. Beams 20, 010401 (2017).

[4] D. V. Rose, E. A. Madrid, D. R. Welch, R. E. Clark, C. B. Mostrom, W. A. Stygar, and M. E. Cuneo, Computational analysis of current-loss mechanisms in a post-hole convolute driven by magnetically insulated transmission lines, Phys. Rev. ST Accel. Beams 18, 030402 (2015).

[5] R. W. Lemke, D. B. Sinars, E. M. Waisman et al., Effects of Mass Ablation on the Scaling of X-Ray Power with Current in Wire-Array Z Pinches, Phys. Rev. Lett. 102, 025005 (2009).

[6] C. A. Jennings, M.E. Cuneo, E. M. Waisman, D. B. Sinars, D. J. Ampleford, G. R. Bennett, W. A. Stygar, and J. P. Chittenden, Simulations of the implosion and stagnation of compact wire arrays, Phys. Plasmas 17, 092703 (2010).

[7] D. H. McDaniel, M. G. Mazarakis, D. E. Blis et al., in Proceedings of the 5th International Conference on Dense Z-Pinches, edited by C. Deeney, N. R. Pereira, and J. Davis (AIP, Melville, NY, 2002), Vol. 651, pp. 23-28.

[8] R. D. McBride, C. A. Jennings, R. A. Vesey et al., Displacement current phenomena in the magnetically insulated transmission lines of the refurbished $\mathrm{Z}$ accelerator, Phys. Rev. ST Accel. Beams 13, 120401 (2010).

[9] L. M. Barker and R. E. Hollenbach, Laser interferometer for measuring high velocities of any reflecting surface, J. Appl. Phys. 43, 4669 (1972).

[10] B. T. Hutsel et al., Transmission-line-circuit model of an 85-TW, 25-MA pulsed-power accelerator, Phys. Rev. Accel. Beams 21, 030401 (2018).

[11] J. Pace VanDevender, T. D. Pointon, D. B. Seidel, K. W. Struve, C. Jennings, B. V. Oliver, and L. X. Schneider, Requirements for self-magnetically insulated transmission lines, Phys. Rev. ST Accel. Beams 18, 030401 (2015).

[12] P. F. Ottinger and J. W. Schumer, Magnetically insulated ion flow theory, Phys. Plasmas 13, 063101 (2006).

[13] H. Wang, L. Meng, D. Liu et al., Magnetically insulated theory with both electron and ion flows, Phys. Plasmas 19, 103506 (2012).

[14] J. W. Schumer, P. F. Ottinger, and C. L. Olson, Power flow in a magnetically insulated recyclable transmission line for a Z-pinch-driven inertial-confinement-fusion energy system, IEEE Trans. Plasma Sci. 34, 2652 (2006).

[15] W. A. Stygar, T. C. Wagoner, H. C. Ives et al., Analytic model of a magnetically insulated transmission line with collisional flow electrons, Phys. Rev. ST Accel. Beams 9, 090401 (2006).

[16] W. A. Stygar, S. E. Rosenthal, H. C. Ives et al., Energy loss to conductors operated at lineal current densities $\leq 10 \mathrm{MA} / \mathrm{cm}$ : Semianalytic model, magnetohydrodynamic simulations, and experiment, Phys. Rev. ST Accel. Beams 11, 120401 (2008); T. W. L. Sanford et al., Measurement of electron energy deposition necessary to form an anode plasma in $\mathrm{Ta}, \mathrm{Ti}$, and $\mathrm{C}$ for coaxial bremsstrahlung diodes, J. Appl. Phys. 66, 10 (1989); M. E. Cuneo et al., Results of vacuum cleaning techniques on the performance of $\mathrm{LiF}$ field-threshold ion sources on extraction applied-B ion diodes at 1-10 TW, IEEE Trans. Plasma Sci. 25, 2 (1997).

[17] C. W. Mendel, Jr., D. B. Seidel, and S. A. Slutz, A general theory of magnetically insulated electron flow, Phys. Fluids 26, 3628 (1983).

[18] M. P. Desjarlais, Impedance characteristics of applied-B ion diodes, Phys. Rev. Lett. 59, 2295 (1987).

[19] M. P. Desjarlais, Theory of applied-B ion diodes, Phys. Fluids B 1, 1709 (1989).

[20] R. C. Davidson, in Physics of Nonneutral Plasmas (AddisonWesley, Reading, MA, 1990), p. 532.

[21] CHICAGO is a hybrid particle in cell code being developed by Voss Scientific, Albuquerque, NM, with partial support from the Defense Advanced Research Projects Agency and the Air Force Office of Scientific Research. 
[22] M. E. Cuneo, IEEE Trans. Dielectrics Electrical Insul. 6, 4 (1999).

[23] E. A. Madrid, D. V. Rose, D. R. Welch et al., Steady-state modeling of current loss in a post-hole convolute driven by high power magnetically insulated transmission lines, Phys. Rev. ST Accel. Beams 16, 120401 (2013).
[24] D. V. Rose, E. A. Madrid, D. R. Welch, R. E. Clark, C. B. Mostrom, W. A. Stygar, and M. E. Cuneo, Computational analysis of current-loss mechanisms in a post-hole convolute driven by magnetically insulated transmission lines, Phys. Rev. ST Accel. Beams 18, 030402 (2015). 